\title{
Topological Insulators by Topology Optimization
}

\section{Christiansen, Rasmus E.; Wang, Fengwen; Sigmund, Ole}

\section{Published in:}

Physical Review Letters

Link to article, DOI:

10.1103/PhysRevLett.122.234502

Publication date:

2019

Document Version

Publisher's PDF, also known as Version of record

Link back to DTU Orbit

Citation (APA):

Christiansen, R. E., Wang, F., \& Sigmund, O. (2019). Topological Insulators by Topology Optimization. Physical Review Letters, 122(23), [234502]. https://doi.org/10.1103/PhysRevLett.122.234502

\section{General rights}

Copyright and moral rights for the publications made accessible in the public portal are retained by the authors and/or other copyright owners and it is a condition of accessing publications that users recognise and abide by the legal requirements associated with these rights.

- Users may download and print one copy of any publication from the public portal for the purpose of private study or research.

- You may not further distribute the material or use it for any profit-making activity or commercial gain

- You may freely distribute the URL identifying the publication in the public portal

If you believe that this document breaches copyright please contact us providing details, and we will remove access to the work immediately and investigate your claim 


\title{
Topological Insulators by Topology Optimization
}

\author{
Rasmus E. Christiansen, ${ }^{*}$ Fengwen Wang, and Ole Sigmund \\ Department of Mechanical Engineering, Solid Mechanics, Technical University of Denmark, \\ Nils Koppels Allé, B. 404, DK-2800 Kgs. Lyngby, Denmark
}

(Received 15 November 2018; published 14 June 2019)

\begin{abstract}
An acoustic topological insulator (TI) is synthesized using topology optimization, a free material inverse design method. The TI appears spontaneously from the optimization process without imposing explicit requirements on the existence of pseudospin-1/2 states at the TI interface edge, or the Chern number of the topological phases. The resulting TI is passive, consisting of acoustically hard members placed in an air background and has an operational bandwidth of $\approx 12.5 \%$ showing high transmission. Further analysis demonstrates confinement of more than $99 \%$ of the total field intensity in the TI within at most six lattice constants from the TI interface. The proposed design hereby outperforms a reference from recent literature regarding energy transmission, field confinement, and operational bandwidth.
\end{abstract}

DOI: 10.1103/PhysRevLett.122.234502

The concept of the topological insulator (TI) stems from condensed matter physics and the quantum spin Hall effect $[1,2]$. Following these seminal works, a growing effort has been dedicated to understanding and designing TIs [3,4], with works demonstrating the engineering of TIs within the fields of photonics [5-12], solid mechanics [13-15], and acoustics [16-20]. This surge in interest is partly fuelled by the incredible promise that TIs can provide backscattering protected, edge-state confined, one-way energy transport, robust under a class of structural defects. Such properties are obviously of broad interest, with numerous applications able to benefit from backscattering protected energy transport, e.g., lasing [21]. Three fundamentally different TI systems are known: time-reversal breaking, time-reversal invariant, and Floquet topological systems, each providing different modes of operation [22]. This Letter considers the timereversal invariant setting in acoustics, allowing for backscattering protected directional energy transport, previously realized by spin-dependent directionally propagating modes, robust towards defects, as illustrated in Fig. 1(a).

Acoustic systems intrinsically possess spin 0; thus, no Kramers doublets exist, hindering the manifestation of the acoustic quantum spin Hall effect. This barrier can be overcome by constructing artificial acoustic spin- $1 / 2$ states, e.g., by creating circulating acoustic waves, actively [17] utilizing airflow, or passively [18] by engineering an accidental double Dirac cone through changing the filling factor of cylindrical metallic rods in a honeycomb lattice. In Ref. [18] a time-reversal invariant acoustic TI is engineered, shown to support topologically protected edge states in a $\approx 1.5 \mathrm{kHz}$ wide bulk band gap, demonstrated to largely suppress backscattering, with measured transmission dropping at most $5 \mathrm{~dB}$, and to perform robustly under geometric defects, showing a maximum transmission drop of $4 \mathrm{~dB}$.
As outlined above, significant effort has been invested in the design of TIs, leading to excellent results and new discoveries. The design procedures have, however, hitherto mainly been based on intuition and the bottom-up approach of band-structure engineering. Such approaches do not consider the finite size of the physical structure, disregarding the coupling into and out of the TI. Further, approaches based on intuition are unlikely to lead to optimal designs, possibly leaving a large performance potential untapped.

Inspired by Ref. [18], this Letter proposes a fundamentally different, optimization-based approach capable of designing topological insulators: a top-down approach based on inverse design where the backscattering protected energy transport is targeted directly, with no explicit requirements on the underlying mechanisms. Hence, the approach does not impose explicit requirements on the preexistence of pseudospin-1/2 edge states, nor on the Chern numbers of the topological phases, nor on band symmetry inversion in reciprocal space. These properties appear spontaneously during the design process. A TI designed using the proposed approach is analyzed and demonstrated to suppress backscattering from geometric defects while facilitating spin-dependent, directional energy transport and strong field confinement.

The proposed approach considers a carefully configured finite material slab, illuminated by an acoustic source, placed in a homogeneous background medium. It utilizes density-based topology optimization [23] to solve the inverse design problem starting from an initial guess provided by the user and is inspired by Ref. [24]. It is noted that while the topology optimization method and the topological insulator share the word "topology," the two uses are not directly related. In topology optimization the word refers to the ultimate spatial design freedom that 


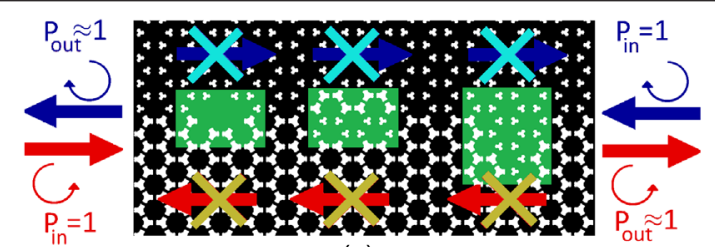

(a)

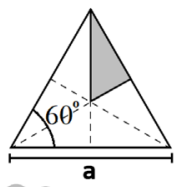

$\Omega_{\mathrm{D}, i} i_{i \in\{1,2\}}$

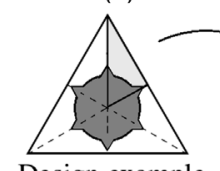

Design example

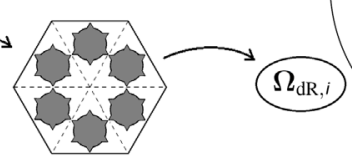

C6v symmetry

(b)

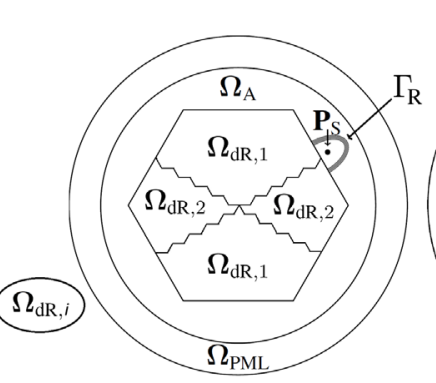

(c)

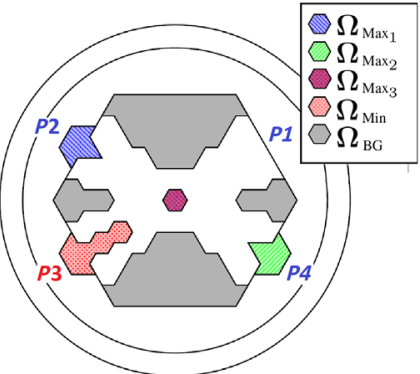

(d)

FIG. 1. (a) Illustration of backscattering protected, pseudospin-dependent directional field propagation through a TI with (green) defects. (b) Left: Design cell showing the (gray) designable region and (dashed lines) mirror symmetry lines. Middle and right: Design example illustrating the symmetry. (c) Model domain $\boldsymbol{\Omega}_{A}$, PML layer $\boldsymbol{\Omega}_{\mathrm{PML}}$, and domains $\boldsymbol{\Omega}_{\mathrm{dR}, 1}$ and $\boldsymbol{\Omega}_{\mathrm{dR}, 2}$ containing the topological phases. Monopolar source $\mathbf{P}_{s}$ in the focal point of a reflector with surface $\Gamma_{R}$. (d) Domains for computing $\Phi_{\mathrm{Total}}$ and $\Phi_{\mathrm{BG}}$ and port numbering, $P 1-P 4$.

allows the algorithm to choose the structural topology which optimizes the objective function. Several recent works have demonstrated the benefit of using topology optimization in the design of exotic metamaterials and crystals, such as multifunctional optical metagratings [25], elastic metamaterials with negative effective material parameters [26], and self-collimating phononic crystals [27]. Further, two review papers [28,29] of inverse design in photonics show numerous successful uses of topology optimization.

A sketch of the model domain serving as the design platform is shown in Fig. 1(c). Here, $\Omega_{A}$ denotes an air region surrounded by a perfectly matched layer [30], denoted $\Omega_{\mathrm{PML}}$. A hexagonally shaped design domain is placed inside $\Omega_{A}$ and partitioned into the subdomains $\Omega_{\mathrm{dR}, 1}$ and $\Omega_{\mathrm{dR}, 2}$ containing two different periodic structures. The slab is illuminated by a monopolar point source $\mathbf{P}_{S}$ placed in the focal point of a perfectly reflecting parabolic reflector $\left[\mathbf{n} \cdot \nabla \Psi(\mathbf{r})=0 \quad \forall \mathbf{r} \in \Gamma_{R}\right]$.

Our fundamental goal is to obtain directional and confined energy transport, in turn obtaining compact acoustic waveguides, robust towards defects. We attack this problem using a top-down approach where we only specify confinement (no propagation in bulk phases) and target the transmission characteristics of a structure composed of two geometrically independent periodic bulk domains [Fig 1(c)]. By inserting a field at port $P 1$, maximizing transmission at ports $P 2$ and $P 4$, and minimizing transmission at port $P 3$, we effectively obtain modes that can propagate in directions where medium 1 is on the right-hand side and medium 2 is on the left-hand side, i.e., directional and hence backscattering protected energy transport. By this systematic approach, we directly maximize transmission and minimize backscattering, in turn bypassing the indirect, bottom-up band-engineering approach used previously. Pseudospin- $1 / 2$ edge states are solutions to this problem; however, we emphasize partly that spin-state solutions are not preimposed and partly that solutions are not imposed to be associated with specific points in the Brillouin zone. Nevertheless, it turns out that the top-down optimization spontaneously converges to solutions similar, albeit much more efficient and with larger bandwidths, to previously suggested bottom-up solutions. In this work, we consider a single specific design problem; however, the formulation is general and can be used to investigate other macro- and microscale setups and symmetries.

The physics is modeled using a Helmholtz-type equation,

$\nabla \cdot\left(C_{1} \frac{\omega_{0}-i c \alpha}{\omega_{0}} \nabla \Psi\right)+C_{2} \frac{\left(\omega_{0}-i c \alpha\right)^{3}}{\omega_{0}} \Psi=-\mathbf{P}_{S}$

where $C_{1}(\mathbf{r})$ and $C_{2}(\mathbf{r})$ are material-dependent parameters, $i$ is the imaginary unit, $\alpha(\mathbf{r})$ is an attenuation parameter, $\omega_{0}=2 \pi f_{0}$ is the free-space angular frequency, $c$ is the free-space wave speed, $\Psi(\mathbf{r})$ is the state field, and $\mathbf{r}$ is the spatial position. For the acoustic case, $\Psi=p$, where $p(\mathbf{r})$ is the sound pressure, and $\left\{C_{1}, C_{2}\right\}=\{(1 / \rho),(1 / \kappa)\}$, where $\rho(\mathbf{r})$ and $\kappa(\mathbf{r})$ are the density and bulk modulus, respectively. Material parameters for air and aluminium are used [31]. The impedance contrast between the two ensuring that vibrations exited in the solid are negligible, and thus Eq. (1) accurately captures the physics, as verified in Refs. [32,33].

The design problem is formulated as a continuous constrained optimization problem and solved using density-based topology optimization. A spatial design field $\xi(\mathbf{r}) \in[0,1] \quad \forall \mathbf{r} \in \Omega_{\mathrm{dR}, 1} \cup \Omega_{\mathrm{dR}, 2}$ is introduced to control the periodic material distributions in $\Omega_{\mathrm{dR}, 1}$ and $\Omega_{\mathrm{dR}, 2}$ by interpolating $C_{1}$ and $C_{2}$ between the material parameters as 
$C_{i}^{-1}(\mathbf{r})=C_{i_{\text {air }}}^{-1}+\xi(\mathbf{r})^{6}\left(C_{i_{\text {aluminium }}}^{-1}-C_{i_{\text {air }}}^{-1}\right), \quad i \in\{1,2\}$.

Figure 1(b) shows the base design cells in which the material distribution is manipulated to solve the optimization problem. The content of each base cell is duplicated throughout $\Omega_{\mathrm{dR}, 1}$ and $\Omega_{\mathrm{dR}, 2}$ to construct the material distribution (topological phases) used when solving Eq. (1). For the example treated in this Letter, $C 3 v$ symmetry is imposed on both base cells. The designable region is colored gray and the mirror symmetries are shown using dashed lines. An example of a design for one phase and its symmetry is illustrated.

The optimization problem is written as

$$
\begin{gathered}
\max _{\xi(\mathbf{r}) \in[0,1]} \Phi_{\mathrm{Total}}(\xi)=\sum_{i=1}^{3} \Phi_{\mathrm{Max}_{i}}(\xi)-\Phi_{\mathrm{Min}}(\xi), \\
\text { s.t. } \Phi_{\mathrm{BG}}(\xi) \leq \gamma_{1} \\
\gamma_{2}<\Phi_{\mathrm{Max}_{1}}(\xi) / \Phi_{\mathrm{Max}_{2}}(\xi)<\gamma_{3}
\end{gathered}
$$

where $\Phi_{\text {Total }}$ is the objective function consisting of a linear combination of the terms $\Phi_{\mathrm{Max}_{i}}, i \in\{1,2,3\}$, and $\Phi_{\mathrm{Min}}$, all of which are integrals of the field intensity magnitude over $\Omega_{\mathrm{Max}_{i}}, i \in\{1,2,3\}$, and $\Omega_{\mathrm{Min}}$, while $\Phi_{\mathrm{BG}}$ denotes the integral of the field intensity magnitude over $\Omega_{\mathrm{BG}}$; see Fig. 1(d). The constants $\gamma_{j}>0, j \in\{1,2,3\}$ control the constraints Eqs. (4) and (5), and $\Phi_{\star}$ is calculated as

$$
\begin{aligned}
\Phi_{\star}(\xi)= & \tau_{\star} \int_{\Omega_{\star}}|I(\Psi(\xi))| d \mathbf{r} / \int_{\Omega_{\star}} d \mathbf{r}, \\
& \star \in\left\{\operatorname{Max}_{i}, \operatorname{Min}, \mathrm{BG}\right\}, \quad i \in\{1,2,3\} .
\end{aligned}
$$

Here $I(\Psi(\xi))$ denotes the field intensity and $\tau_{\star}$ a set of scaling constants. The choice of $\Phi_{\text {Total }}$ leads to a maximization of the energy transmitted into $\Omega_{\mathrm{Max}_{1}}$ and $\Omega_{\mathrm{Max}_{2}}$ along with a simultaneous minimization of the energy transmitted into $\Omega_{\text {Min }}$. That is, in order to maximize $\Phi_{\text {Total }}$, any field emitted by $\mathbf{P}_{S}$, propagating along the interface between $\Omega_{\mathrm{dR}, 1}$ and $\Omega_{\mathrm{dR}, 2}$, must keep $\Omega_{\mathrm{dR}, 1}$ on its right-hand side and $\Omega_{\mathrm{dR}, 2}$ on its left-hand side at all times. This in turn promotes backscattering protected transport of energy along the interface. The constraint Eq. (4) ensures that a bulk band gap exists in both topological phases, as energy is prohibited from propagating into $\Omega_{\mathrm{BG}}$. The constraint Eq. (5) may be used to control the ratio of the intensity transmitted to $\Phi_{\mathrm{Max}_{1}}$ and $\Phi_{\mathrm{Max}_{2}}$, respectively.

The design problem, Eqs. (1)-(6), is implemented and solved in COMSOL MULTIPHYSICS 5.3 using the globally convergent method of moving asymptotes [34] to solve Eqs. (3)-(5). The objective function gradients are calculated using adjoint sensitivity analysis [28]. A physically admissible final design, consisting solely of solid and air and free of

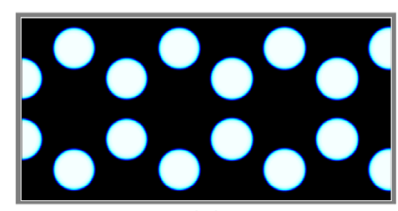

(a)

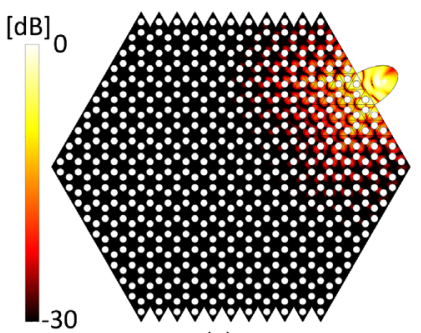

(c)

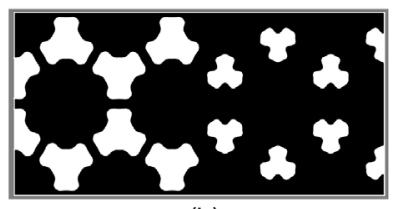

(b)

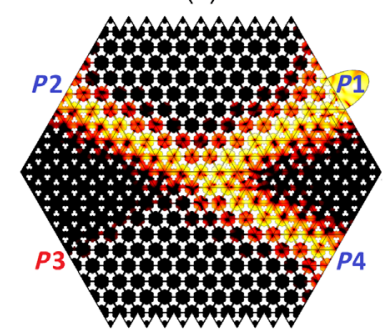

(d)

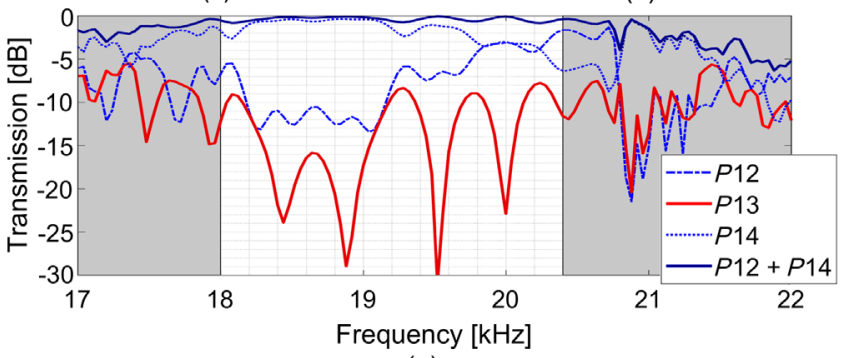

(e)
FIG. 2. (a) Initial and (b) optimized material configuration in supercells consisting of the two topological phases (black) air, (white) solid, (shades of blue) air and solid mixture. (c),(d) Sound pressure at $f_{0}=20 \mathrm{kHz}$ for the (c) initial and (d) optimized material distribution in $\Omega_{\mathrm{dR}, 1} \cup \Omega_{\mathrm{dR}, 2}$. Color map: Pressure magnitude, (white) solid material. (d) The targeted backscattering protected edge-state energy transport is observed. (e) Transmission to ports $P 2, P 3$, and $P 4$, as a function of frequency, normalized to the energy flowing through port $P 1$. [Port numbering in (d).]

numerical artifacts, is assured using the projection and filtering procedure outlined in Refs. [24,35,36].

For the TI considered in the following, Eqs. (1)-(6) are solved with $\left\{a=0.01 \mathrm{~m}, f_{0}=20 \mathrm{kHz}, c=343 \mathrm{~m} / \mathrm{s}\right.$, $\alpha=6 \mathrm{~dB} / \lambda \forall \mathbf{r} \in \Omega_{A}, \alpha=0 \mathrm{~dB} / \lambda \forall \mathbf{r} \in \Omega_{\mathrm{dR}, 1} \bigcup \Omega_{\mathrm{dR}, 2}$, $\gamma_{1}=0.04, \gamma_{2}=0.3, \gamma_{3}=1.7, \tau_{\mathrm{Max}_{1}}=1, \tau_{\mathrm{Max}_{2}}=1, \tau_{\mathrm{Max}_{3}}=$ $\left.0.1, \tau_{\mathrm{Min}}=4, \tau_{\mathrm{BG}}=1\right\}$. The initial $\xi(\mathbf{r})$ layout, shown in Fig. 2(a), is chosen to constitute a crystal with a bulk band gap at $f_{0}$ [see the band structure in Fig. 3(a)]. The final material layout obtained from the optimization process is shown in Fig. 2(b), with white (black) representing solid (air).

The max-normalized pressure field at $f_{0}=20 \mathrm{kHz}$ along with the initial and optimized material configurations in $\Omega_{\mathrm{dR}, 1} \cup \Omega_{\mathrm{dR}, 2}$ are shown in Figs. 2(c) and 2(d), respectively. The bulk band gap of the initial material configuration is observed. For the optimized TI it is clear that the vast majority of the energy flowing into port $P 1$ is transmitted to either port $P 2$ or port $P 4$. Simultaneously, a bulk band gap is observed for both phases of the TI. Figure 2(e) presents a frequency sweep of the transmission 


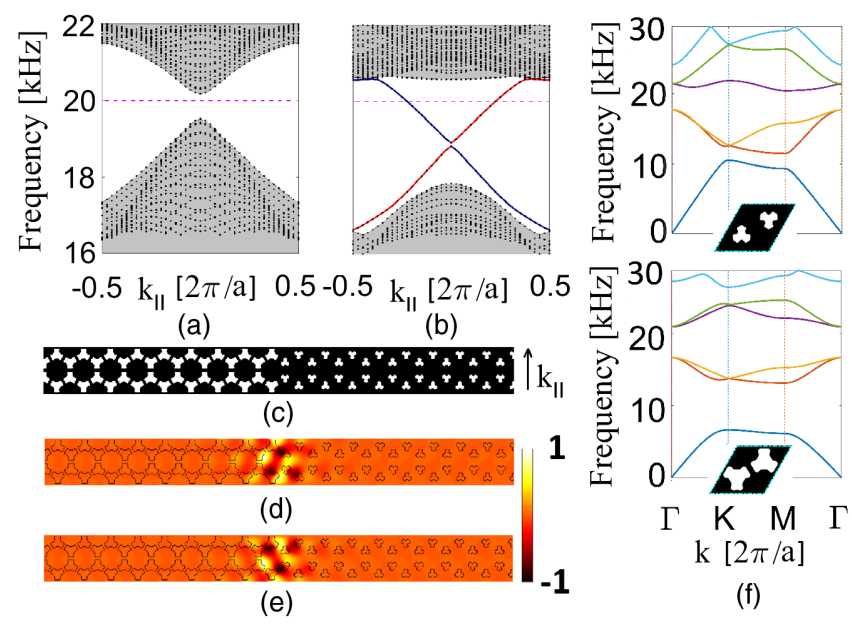

FIG. 3. Supercell band structure for the (a) initial material configuration and (b) optimized TI design. The edge-state bands are colored indicating (red) positive, and (blue) negative pseudospin-1/2, and (white) bulk band gap. (c) Supercell used to compute (b). (d), (e) Normalized eigenmodes at $f_{0}=20 \mathrm{kHz}$. (f) Band structures for the two crystal phases.

to $P 2, P 3$, and $P 4$, normalized to the power flowing through $P 1: 10 \log _{10}\left(\left|P_{x}\right| /\left|P_{1}\right|\right)$. It is seen that $99.5 \%$ of the acoustic power is transmitted from $P 1$ to $P 2$ and $P 4$ at $f_{0}=20 \mathrm{kHz}$. Further, the transmission does not drop below $-0.85 \mathrm{~dB}$ from 18 to $20.4 \mathrm{kHz}$.

The above discussion demonstrates that the top-down approach results in the desired macroscopic response. However, this could in principle be attained without having designed a TI. That a TI has indeed appeared spontaneously through the optimization process is revealed in the following analysis.

Figure 3(b) shows the band structure diagram, calculated for the TI supercell shown in Fig. 3(c) using periodic boundary conditions on the top and bottom edges and Neumann conditions on the left and right edges. The bulkband regions are colored gray and the two "crossing" symmetry inverted edge-state bands are colored red and blue corresponding to the positive or negative pseudospin- $1 / 2$ edge states, shown for $f_{0}=20 \mathrm{kHz}$ in Figs. 3(d) and 3(e), respectively. From Fig. 3(b) the bulk band gap is seen to stretch from $\approx 18$ to $\approx 20.4 \mathrm{kHz}$, i.e., $\approx 12.5 \%$. A narrow gap is seen in the two edge-state bands at the $k_{\|}=0$ point. A similar gap was reported in Ref. [18], where it was explained to originate from the imperfect cladding layer. Figure 3(f) shows the band structures for the two crystal phases constituting the TI, revealing degeneracies for bands 2 and 3 and bands 4 and 5 at the $\Gamma$ point for both phases.

To further investigate if a TI supporting geometrically robust backscattering protected transport of acoustic energy has been designed, studies on the effect of introducing defects in the TI are performed. Figure 4 presents four examples, with Fig. 4(a) showing a slab without defects,

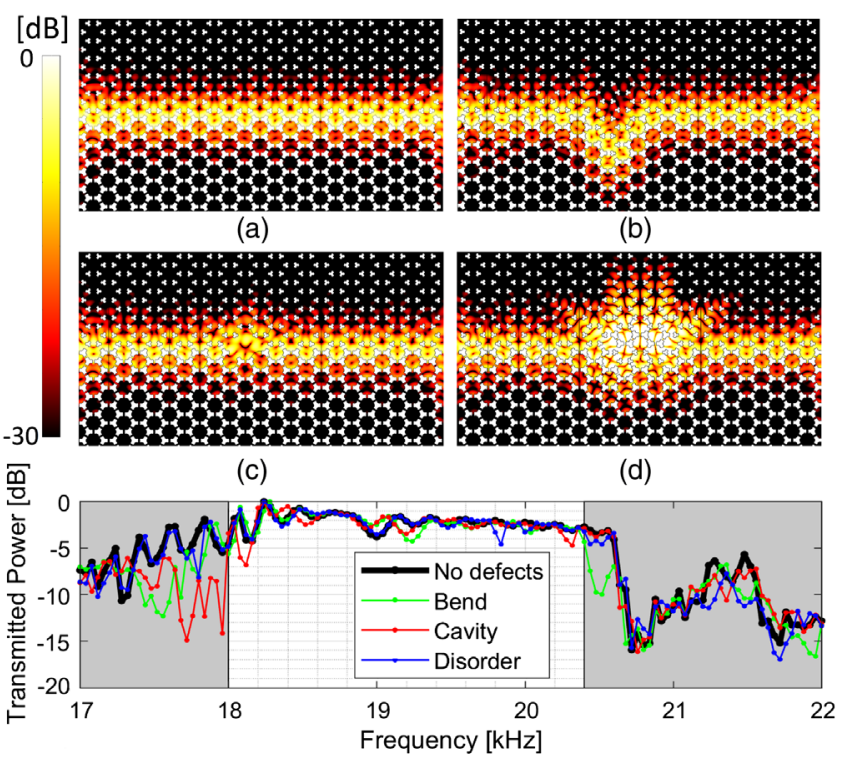

(e)

FIG. 4. Investigation of robust energy transport in TI edge state under geometric defects. (a)-(d) Color map: Max-normalized sound pressure at $20 \mathrm{kHz}$, (white) solid material. (a) Unperturbed TI. (b) TI with bend. (c) TI with cavity. (d) TI with disorder. (e) Max-normalized transmitted power, recorded after the material slab, for the configurations shown in (a)-(d).

while Figs. 4(b)-4(d) show a bend, cavity, and disorder defect, respectively. The three defects all preserve the symmetry of the bulk materials and are shown in Fig. 1(a) (highlighted green). The slabs are excited by a point source positioned $0.3 a$ from their left edge, at the interface of the two topological phases. The power, transmitted through the TI, is computed at the right-hand side of the slab and the results are reported in Fig. 4(e), max normalized with respect to the nondefect TI.

Figure 4(e) reveals agreement of the transmitted power inside the bulk band gap across the four cases. The largest deviation between the nondefect and defect structures is $2.5 \mathrm{~dB}$, and intervals showing less than $0.25 \mathrm{~dB}$ deviation are observed supporting that a TI offering backscattering protected propagation has been designed. The differences in transmission seen in Fig. 4(e) are orders of magnitude smaller than the differences observed across the majority of the band of operation for similar defects in a traditional phononic crystal waveguide, with a worst-case value of more than $25 \mathrm{~dB}$ reported in Ref. [18].

An important aspect to consider when designing systems for energy or information transport, such as waveguides, is the footprint of the system. In the present context, the footprint refers to how wide the material slab must be to confine a certain fraction of the transported energy. From Fig. 4(a), the pressure field in the TI appears to be confined (to a 30-dB level) inside approximately $3 a$ from the TI interface edge. An investigation of the spatial confinement of the field is performed using the TI from Ref. [18] as a 


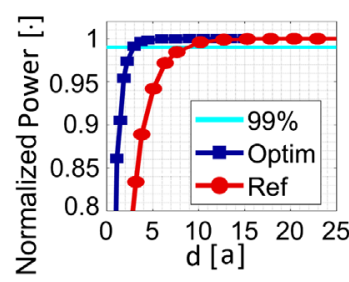

(a)

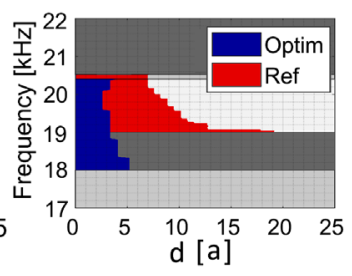

(b)

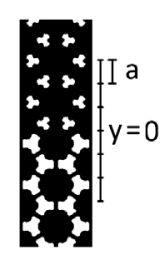

(c)
FIG. 5. Spatial field confinement of the TI edge state. (a) Fraction of the total power contained within a distance $d$ from the TI interface edge $P_{f}(d)$, at $f_{0}=20 \mathrm{kHz}$, for [Optim] the proposed TI design and [Ref] the TI in Ref. [18]. Map of the distance $d$ versus frequency, within which more than $99 \%$ of the total power is confined, $P_{f}(d)>0.99$ for [Optim] the optimized TI design and [Ref] the reference. (c) TI supercell, (white) solid material, (black) air.

reference. This is done by calculating the fraction of the total power flowing through the TI within a distance $d$ from the TI interface edge [see the illustration in Fig. 5(c)] as $P_{f}(d)=\int_{-d}^{d} \mathbf{n} \cdot \mathbf{I}(y) d y / \int_{-\infty}^{\infty} \mathbf{n} \cdot \mathbf{I}(y) d y, d>0$.

The results for $f_{0}=20 \mathrm{kHz}$, shown in Fig. 5(a), reveal that more than $99 \%$ of the power is contained within $d \approx 3 a$ for the TI design proposed in this Letter, while for the reference design [18] $d \approx 9 a$ is required. A map showing the distance $d$ within which $99 \%$ or more of the power is confined versus frequency for both TIs is provided in Fig. 5(b). The map reveals that at most a distance of $6 a$ is required to contain $99 \%$ of the power for the proposed TI.

In summary, this Letter reports on the design of a topological insulator using a top-down approach based on density-based topology optimization. The approach directly targets the desired effect of backscattering protected, directional energy transport. That the effect is achieved by the resulting TI is demonstrated through numerical studies. Experimental validation of the approach may be found in Ref. [33], where a metamaterial exhibiting negative refraction is considered.

The proposed design approach is trivially extendable to photonics, assuming TE or TM polarized light. Further, by introducing additional design constraints and goals it is straightforwardly extendable to, e.g., consider global defects in the TI or to target a maximization of the operational bandwidth in the design process. Hence, the approach has freedom to tailor TIs to operate under alternative conditions.

The authors acknowledge discussions with S. Stobbe and support from NATEC (NAnophotonics for TErabit Communications) Centre (Grant No. 8692) which is awarded by Villum Fonden.

*Corresponding author. raelch@mek.dtu.dk
[1] D. J. Thouless, M. Kohmoto, M. P. Nightingale, and M. den Nijs, Phys. Rev. Lett. 49, 405 (1982).

[2] F. D. M. Haldane, Phys. Rev. Lett. 61, 2015 (1988).

[3] M. Z. Hasan and C. L. Kane, Rev. Mod. Phys. 82, 3045 (2010).

[4] X. L. Qi and S. C. Zhang, Rev. Mod. Phys. 83, 1057 (2011).

[5] A. B. Khanikaev, S. H. Mousavi, W.-K. Tse, M. Kargarian, A. H. MacDonald, and G. Shvets, Nat. Mater. 12, 233 (2013).

[6] S. Raghu and F. D. M. Haldane, Phys. Rev. A 78, 033834 (2008).

[7] Z. Wang, Y. D. Chong, J. D. Joannopoulos, and M. Soljačić, Phys. Rev. Lett. 100, 013905 (2008).

[8] Z. Wang, Y. D. Chong, J. D. Joannopoulos, and M. Soljačić, Nature (London) 461, 772 (2009).

[9] M. Hafezi, E. A. Demler, M. D. Lukin, and J. M. Taylor, Nat. Phys. 7, 907 (2011).

[10] W. Chen, S. Jiang, X. Chen, B. Zhu, L. Zhou, J. Dong, and C. Chan, Nat. Commun. 5, 5782 (2014).

[11] L. Lu, J. D. Joannopoulos, and M. Soljačić, Nat. Photonics 8, 821 (2014).

[12] L.-H. Wu and X. Hu, Phys. Rev. Lett. 114, 223901 (2015).

[13] R. Süsstrunk and S. D. Huber, Science 349, 47 (2015).

[14] P. Wang, L. Lu, and K. Bertoldi, Phys. Rev. Lett. 115, 104302 (2015).

[15] S. H. Mousavi, A. B. Khanikaev, and Z. Wang, Nat. Commun. 6, 8682 (2015).

[16] X. Ni, C. He, X. Sun, X. Liu, L. Feng, and Y. Chen, New J. Phys. 17, 053016 (2015).

[17] Z. Yang, F. Gao, X. Shi, X. Lin, Z. Gao, Y. Chong, and B. Zhang, Phys. Rev. Lett. 114, 114301 (2015).

[18] C. He, X. Ni, H. Gu, X. Sun, Y. Chen, M. Lu, X. Liu, and Y. Chen, Nat. Phys. 12, 1124 (2016).

[19] A. B. Khanikaev, R. Fleury, S. H. Mousavi, and A. Alu, Nat. Commun. 6, 8260 (2015).

[20] R. Fleury, A. B. Khanikaev, and A. Alu, Nat. Commun. 7, 11744 (2016).

[21] P. St-Jean, V. Goblot, A. Lemaître, T. Ozawa, L. L. Gratiet, I. Sagnes, J. Bloch, and A. Amo, Nat. Photonics 11, 651 (2017).

[22] A. B. Khanikaev and G. Shvets, Nat. Photonics 11, 763 (2017).

[23] M. P. Bendsøe and O. Sigmund, Topology Optimization (Springer, New York, 2003).

[24] R. E. Christiansen and O. Sigmund, Struct. Multidiciplinary Optim. 54, 469 (2016).

[25] D. Sell, J. Yang, S. Doshay, R. Yang, and J. A. Fan, Nano Lett. 17, 3752 (2017).

[26] H.-W. Dong, S.-D. Zhao, Y.-S. Wang, and C. Zhang, J. Mech. Phys. Solids 105, 54 (2017).

[27] J. H. Park, P. S. Ma, and Y. Y. Kim, Struct. Multidiscip. Optim. 51, 1199 (2015).

[28] J. S. Jensen and O. Sigmund, Laser Photonics Rev. 5, 308 (2011).

[29] S. Molesky, Z. Lin, A. Y. Piggott, W. Jin, and J. V. Alejandro, and W. Rodriguez, Nat. Photonics 12, 659 (2018).

[30] J.-P. Berenger, J. Comput. Phys. 114, 185 (1994). 
[31] M. B. Dühring, J. S. Jensen, and O. Sigmund, J. Sound Vib. 317, 557 (2008).

[32] R. E. Christiansen, E. Fernandez-Grande, and O. Sigmund, J. Acoust. Soc. Am. 138, 3470 (2015).

[33] R. E. Christiansen and O. Sigmund, Appl. Phys. Lett. 109, 101905 (2016).
[34] K. Svanberg, SIAM J. Optim. 12, 555 (2002).

[35] F. Wang, B. S. Lazarov, and O. Sigmund, Struct. Multidiciplinary Optim. 43, 767 (2011).

[36] J. K. Guest, J. H. Prévost, and T. Belytschko, Int. J. Numer. Methods Eng. 61, 238 (2004). 